\title{
The Estimation of the Fat Free Mass Index in Athletes
}

Jeremy P. Loenneke, MSc; Jacob M. Wilson, PhD; Mandy E. Wray, MSc; Jeremy T. Barnes PhD; Monica L. Kearney MS; Thomas J. Pujol, EdD

\author{
Authors' Affiliation: \\ 1. Department of Health and Exercise \\ Science, The University of \\ Oklahoma, Norman, USA \\ 2. Department of Health Sciences and \\ Human Performance, University of \\ Tampa, Tampa, USA \\ 3. Department of Nutritional Sciences, \\ The University of Oklahoma Health \\ Sciences Center, Oklahoma City, \\ USA \\ 4. Department of Health, Human \\ Performance, and Recreation. \\ Southeast Missouri State University, \\ Cape Girardeau, USA \\ 5. Department of Nutrition and \\ Exercise Physiology, University of \\ Missouri, Columbia, USA \\ * Corresponding Author; \\ Address: 1401 Asp Ave., Room 104. \\ Norman, Oklahoma 73019-0615, USA \\ E-mail: jploenneke@ou.edu
}

Received: Mar 09, 2012

Accepted: Jun 24, 2012

Key Words: Body Mass Index; Body Fat; Sports; Fat Free Mass; Dual Energy X-ray Absorptiometry; DEXA Scan

\begin{abstract}
Purpose: The purpose of this investigation was to compare a practical measurement of fat free mass index (FFMI) from bioelectrical impedance analysis (BIA) to the dual energy X-ray absorptiometry (DEXA) value in collegiate athletes.

Methods: Thirty-three male baseball players and 16 female gymnasts volunteered to participate in this study during their respective pre-season. Subjects visited the laboratory once and had their measurements taken in the following order: weight, height, DEXA, and Omron HBF-500.

Results: The BIA device investigated was not a valid estimate of FFMI when compared to the DEXA. The TE was $0.93 \mathrm{~kg} / \mathrm{m}^{2}$ for males and $0.78 \mathrm{~kg} / \mathrm{m}^{2}$ for females. There were also significant mean differences between the BIA prediction and the DEXA value for males $\left(B I A=20.6 \mathrm{~kg} / \mathrm{m}^{2} \mathrm{vs}\right.$. DEXA $=21.1$ $\left.\mathrm{kg} / \mathrm{m}^{2}, P=0.007\right)$ and females $\left(B I A=16.2 \mathrm{~kg} / \mathrm{m}^{2}\right.$ vs. DEXA $\left.=17.5 \mathrm{~kg} / \mathrm{m}^{2}, P=0.001\right)$.

Conclusions: The BIA device investigated in this study did not provide a valid estimate of FFMI in male and female collegiate athletes. Although there was a general tendency for the BIA to underestimate FFMI compared to DEXA, $98 \%$ of the estimates were within plus or minus $2 \mathrm{~kg} / \mathrm{m}^{2}$. Therefore, while slightly biased, BIA may provide a reasonable $\left( \pm 2 \mathrm{~kg} / \mathrm{m}^{2}\right)$ estimate of nutritional status for practitioners who are unable able to afford more expensive equipment.
\end{abstract}

Asian Journal of Sports Medicine, Volume 3 (Number 3), September 2012, Pages: 200-203

\section{INTRODUCTION}

$\mathrm{W}$ hen assessing the fitness levels of athletes, body composition is usually estimated. In addition, it is also important to know the body composition of your athletes from a nutritional standpoint. Nutritional status is typically determined from the use of body mass index (BMI), however there are numerous cases where BMI may not accurately reflect the actual composition of the athlete ${ }^{[1]}$. Fat free mass index (FFMI) might offer a better representation since it includes an actual estimate of body composition in the equation $\left(\text { FFMI }=F F M(\mathrm{~kg}) / \mathrm{m}^{2}\right)^{[2]}$. However, while FFMI might provide a better index of nutritional status ${ }^{[3]}$, it still requires equipment that might not be available to all practitioners in the field. Therefore the purpose of this investigation was to compare a more practical measurement of FFMI from bioelectrical impedance analysis (BIA) to the dual energy X-ray absorptiometry (DEXA) value in collegiate athletes (National Collegiate Athletic Association, Division 1).

\section{METHODS AND SUBJECTS}

\section{Participants:}

Thirty-three male (age: 20 (1) years; height: $1.8(0.1)$ 
m; body mass: 88.2 (9.6) kg; BMI: $26.7(2.1) \mathrm{kg} / \mathrm{m}^{2}$ ) baseball players and 16 female (age: 20 (1) years; height: 1.6 (0.1) m; body mass: 59.4 (5.0) kg; BMI: $\left.22.9(1.7) \mathrm{kg} / \mathrm{m}^{2}\right)$ gymnasts volunteered to participate in this study. Subjects were recruited from the University's baseball and gymnastics team during their respective pre-season. In addition, all data was collected during the same time period. The study was designed to investigate the validity of BIA on estimating FFMI when compared to the DEXA estimate. Subjects visited the bone density laboratory once and had their measurements taken in the following order: weight, height, DEXA, and Omron HBF-500. Subjects were asked to abstain from food and water for 4 hours prior to their visit but understood the importance of promoting hydration before the 4 hour abstinence period; however neither dietary nor hydration status were quantified. Subjects were informed about the procedures and potential risks of the tests before their informed consent was obtained. The University's institutional review board approved this study.

\section{Protocol:}

Dual energy X-ray absorptiometry: The subject's criterion body composition was estimated using a GE Lunar Prodigy DEXA machine (GE Healthcare, Pewaukee, WI). Each day before testing, a quality assurance phantom was performed and passed. Before each test, the subjects' height was measured to the nearest $\mathrm{cm}$ using a wall-mounted stadiometer and body mass was measured using an electronic scale. Subjects were asked to wear shorts and a t-shirt and to remove any objects containing metal. Subjects lain supine on the DEXA table with their hands lying flat and pronated and asked to remain motionless while their body was scanned. Lunar software algorithms calculated the estimates of BF\% for each subject. Fat free mass (FFM) in $\mathrm{kg}$ was determined from the following equation (FFM= Body mass- (Body mass $\mathrm{x}$ DEXA BF\%)). Additionally, FFMI was determined from the equation $\left(\mathrm{FFMI}=\mathrm{FFM}(\mathrm{kg}) / \mathrm{m}^{2}\right)$.

Bioelectrical Impedance Analysis: An Omron HBF500 (leg-to-leg/arm-to-arm) (Omron Healthcare, Kyoto, Japan) was used for BIA measurements according to manufacturer guidelines. Hydration status is important for accurate assessment of body composition using BIA ${ }^{[4]}$. Hence, in accordance with BIA guidelines the subjects were asked to: avoid exercise prior to their test and avoid alcohol the night before their test. In addition subjects were asked not to drink any fluid 4 hours prior to their test and urinate within 30 minutes of test. FFM in $\mathrm{kg}$ was determined from the following equation (FFM= Body mass- (Body mass x BIA BF\%)).

\section{Statistical Analyses:}

The validity of the FFMI estimate was based on the evaluation of the BMI estimated value from the DEXA by calculating the mean, SD, Pearson correlation, and standard error of estimate (SEE) from linear regression analysis. SEE represents the degree of deviation of individual scores from the regression line. To assess the average deviation of individual scores from the line of identity, total error (TE) was calculated for each field method. Paired t-tests determined pair-wise differences between measurements using an alpha level of 0.05. Differences between each method were plotted in Excel (Microsoft, Redmond, WA, USA) against their group mean [(BIA mean+DEXA mean)/2] to determine the directional bias of the BIA estimate. All analyses were made using PASW Statistics 18, with all variability represented using standard deviation (SD).

Table 1: The validity calculations comparing the BIA and DEXA estimates of the FFMI separated by gender

$\begin{array}{lccccc}\text { Gender } & \text { Variable } & \text { FFMI }\left(\mathrm{kg} / \mathrm{m}^{2}\right) & \begin{array}{c}\text { Pearson's } \\ \text { correlation }(\boldsymbol{r})\end{array} & \begin{array}{c}\text { standard error of } \\ \text { the estimate }\left(\mathbf{k g} / \mathbf{m}^{2}\right)\end{array} & \begin{array}{c}\text { Total error } \\ \left(\mathbf{k g} / \mathbf{m}^{2}\right)\end{array} \\ \text { Male } & \begin{array}{c}\text { DEXA } \\ \text { BIA }\end{array} & \begin{array}{c}21.1(1.6) \\ 20.6(1.1)^{*}\end{array} & 0.722 & 1.0 & 0.9 \\ \text { Female } & \begin{array}{c}\text { DEXA } \\ \text { BIA }\end{array} & \begin{array}{c}17.5(0.6) \\ 16.2(0.4)^{*}\end{array} & 0.599 & 0.5 & 0.7\end{array}$

BIA: Bioelectrical Impedance Analysis; DEXA: Dual Energy X-Ray Absorptiometry; FFMI: Estimates of the Fat Free Mass Index * represents a significant mean difference from the DEXA estimate, $P<0.05$ 


\section{RESULTS}

BIA significantly underestimated FFMI for both males (mean difference $=0.51 \mathrm{~kg} / \mathrm{m}^{2}, P=0.007$ ) and females (mean difference $=1.2 \mathrm{~kg} / \mathrm{m}^{2}, P=0.001$ ). The Pearson's correlation, SEE, and TE for each gender is reported in Table 1.

Seventy percent of males and $100 \%$ of females' FFMI was underestimated from BIA when compared to the DEXA (Fig. 1).

\section{DISCUSSION}

Results of this study indicate that the BIA device investigated was not a valid estimate of FFMI when compared to the DEXA for males or females. This is based on the significant differences observed between the BIA prediction and the DEXA value. Figure 1 shows that the BIA underestimated $77 \%$ of the athletes' FFMI. To our knowledge, this is the first investigation determining the validity of a BIA estimates for FFMI in male and female collegiate athletes.

Although BMI has been associated with nutritional status, this simple calculation provides problems for certain populations who might carry more weight in lean tissue (i.e. athletes) ${ }^{[1]}$. When assessing the nutritional status of athletes, it may misclassify those with greater FFM as being obese which makes it difficult to quantify their nutritional status. FFMI may serve as a better index; however, the BIA device used in this study underestimated most male athletes and all of the female athletes. Nevertheless, $98 \%$ of the estimates were within plus or minus $2 \mathrm{~kg} / \mathrm{m}^{2}$. Therefore, although there is a bias to underestimate with this BIA device, it may provide a fairly reasonable snapshot into the current state of the athlete.

Bosy-Westphal et al ${ }^{[5]}$ have previously shown that this BIA device provides a valid estimate of \%BF when compared with the DEXA estimate. However, their study used older males and females ( 45 years). Therefore it is currently unknown if this device can provide a non-biased estimate of FFMI in younger non-

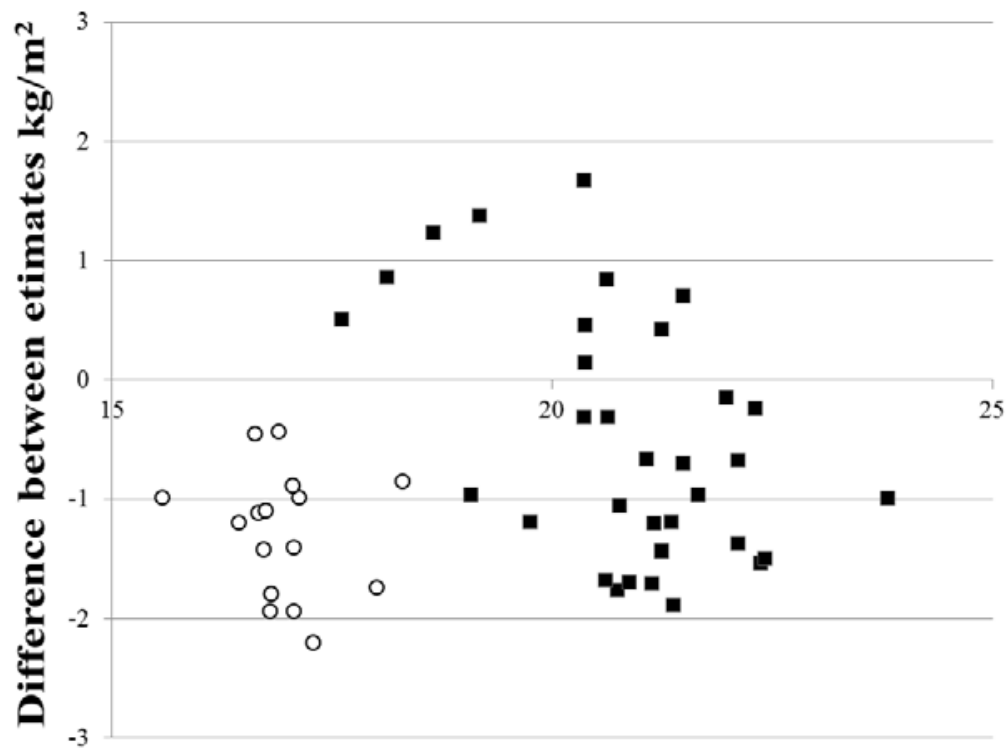

Fig. 1: Differences in fat free mass index between the bioelectrical impedance analysis (BIA) and the dual energy X-ray absorptiometry (DEXA) were plotted against their group mean ((BIA mean+DEXA mean)/2) to determine the directional bias of the BIA estimate compared to the DEXA. Black filled in squares represent male values and black circles represent female values. 
athletic cohorts when compared to the DEXA value. No other published studies to our knowledge have investigated the validity of this BIA device.

Noted limitations of this study include the use of DEXA as our criterion method for estimating body composition because it is not recognized as a gold standard (i.e. underwater weighing) method. In addition, subjects were encouraged to be hydrated however no quantitative measure of hydration was taken. Dehydration would have violated the assumptions made by the DEXA and BIA for estimating body composition. Although a limitation, it also provides a strength in that these devices are often used in settings in which hydration can only be assumed (e.g. outside a laboratory setting). Practitioners realize that although they can give athletes directions to be followed prior to any type of physical fitness assessments there may be wide discrepancies in terms of how well athletes would follow them.

\section{CONCLUSION}

In conclusion, the BIA device investigated in this study did not provide a valid estimate of FFMI in male and female collegiate athletes. Although there was bias for the BIA to underestimate FFMI compared to DEXA, $98 \%$ of the estimates were within plus or minus $2 \mathrm{~kg} /$ $\mathrm{m}^{2}$. Therefore, while slightly biased, BIA may provide a reasonable $\left( \pm 2 \mathrm{~kg} / \mathrm{m}^{2}\right)$ estimate of nutritional status for practitioners who are unable to afford more expensive equipment.

\section{ACKNOWLEDGMENTS}

This manuscript was not supported by any funding.

Conflict of interests: None

\section{REFERENCES}

1. Garn SM, Leonard WR, Hawthorne VM. Three limitations of the body mass index. Am J Clin Nutr 1986;44:996-7.

2. VanItallie TB, Yang MU, Heymsfield SB, et al. Height-normalized indices of the body's fat-free mass and fat mass: potentially useful indicators of nutritional status. Am J Clin Nutr 1990;52:953-9.

3. Schutz Y, Kyle UU, Pichard C. Fat-free mass index and fat mass index percentiles in Caucasians aged 18-98 y. Int J Obes Relat Metab Disord 2002;26:953-60.

4. Heyward VH, Wagner DR. Applied body composition assessment. $2^{\text {nd }}$ ed. Champaign, IL: Human Kinetics. 2004.

5. Bosy-Westphal A, Later W, Hitze B, et al. Accuracy of bioelectrical impedance consumer devices for measurement of body composition in comparison to whole body magnetic resonance imaging and dual X-ray absorptiometry. Obes Facts 2008;1:319-24. 